\title{
THE ELECTRON CLOUD INSTABILITY: SUMMARY OF MEASUREMENTS AND UNDERSTANDING
}

\author{
F. Zimmermann, CERN, Geneva, Switzerland
}

\begin{abstract}
Electron-cloud effects presently limit the performance of several accelerators operating with high beam current, notably the SLAC and KEK B factories, the CERN SPS, the CERN PS, and the Los Alamos PSR. They are a major concern for many future projects, e.g., the CERN LHC and the SNS. An electron cloud is generated in the vacuum chamber by photoemission or beam-induced multipacting and subsequent electron accumulation during a bunch or bunch-train passage. Both coupled and single bunch instabilities, pressure rise, malfunctioning of beam diagnostics and failures of multi-bunch feedback systems have all been attributed to the cloud electrons. We compare observations from various laboratories with computer simulations and analytical estimates, and we address mechanisms by which the electrons may dilute the beam emittance. Possible cures and future research directions are also discussed.
\end{abstract}

\section{INTRODUCTION}

In the early days of the CERN ISR, coupled oscillations of protons and trapped electrons hindered the high-current coasting beam operation [1]. Also at the ISR, after installation of an aluminium test chamber in 1977, beaminduced multipacting was observed with bunched beams, manifesting itself as an orbit-dependent pressure rise [2]. Since 1988, a fast vertical instability accompanied by beam loss limits the maximum current at the Los Alamos PSR, both with bunched and unbunched beam. It was attributed to coupled electron-proton oscillations [3]. A peculiar multi-bunch instability was seen at the KEK photon factory when positron-beam operation started in 1989. Observations here included an increased vertical beam size, coupled oscillation, low threshold current, broad distributions of sidebands, and the inefficiency of a clearing gap. In 1995, this instability was explained by bunch-to-bunch coupling via photoelectrons [4, 5]. From 1996 onwards a series of electron-cloud experiments was performed by an IHEP-KEK collaboration at BEPC [6]. Shortly thereafter, in 1997, crash programs were launched for PEP-II [7, 8] (simulations, TiN coating of $\mathrm{Al}$ vacuum chamber, etc.) and for the LHC [9, 10, 11, 12]. Equally in 1997, an anomalous multi-bunch instability at CESR could be explained by photo-electrons trapped in pump leakage fields [13]. Since 1998, electron-cloud effects are seen with the LHC proton test beam in the SPS, and since 2000 in the CERN PS prior to beam extraction, and even during a single passage in the PS-to-SPS transfer line [14]. The SPS observations include beam loss and emittance growth, presumably caused by coupled-bunch motion in the horizontal plane and by a single-bunch instability in the vertical. Above a certain threshold current, the positron rings of PEP-II and KEKB show a beam-size blow up and a drop in specific luminosity. The onset of blow up depends on the fill pattern. In the complete absence of coherent bunch-to-bunch motion, it must be due to a single-bunch effect.

\section{BUILD UP, SATURATION, DECAY}

In positron and proton storage rings, electrons generated by photo-emission, ionization and secondary emission can accumulate in the beam pipe during multi-bunch operation with close spacing, giving rise to an 'electron cloud'.

Electrons from gas ionization are produced at a rate $d^{2} \lambda_{e} /(d t d s)=v_{p} / c(I / e) \sigma_{\text {ion }} P /\left(k_{B} T\right)$, where $\lambda_{e}$ is the electron line density, $I$ the beam current, $v_{p}$ the beam velocity, $k_{B}$ Boltzmann's constant, $P$ the pressure, and $\sigma_{\text {ion }} \approx 2$ Mbarn the ionization cross section. With $I \approx 1$ A, $P=10$ nTorr, $v_{p} \approx c$ and $T=300 \mathrm{~K}$, we obtain $d^{2} \lambda_{e} /(d t d s) \approx 4 \times 10^{9} \mathrm{~m}^{-1} \mathrm{~s}^{-1}$.

The production of photoelectrons depends on the synchrotron radiation, the reflectivity of the vacuum-chamber surface, and the photo-emission yield. The mean number of synchrotron radiation photons emitted per unit length is [15] $d N_{\gamma} / d s=5 /(2 \sqrt{3}) \gamma / 137 / \rho$, where $\rho$ denotes the bending radius. The photo-emission yield per absorbed photon $Y^{*}$ is of the order 0.1 (for the LHC, special care is taken to obtain $Y^{*} \approx 0.02$ ). Taking typical numbers $\rho \approx 1 \mathrm{~km}, \gamma=10^{6}$ and $Y^{*}$, about one photo-electron is emitted per positron or proton and per meter. Then, for a beam current of order $0.7 \mathrm{~A}$, photoelectrons are produced at a rate $5 \times 10^{18} \mathrm{~m}^{-1} \mathrm{~s}^{-1}$, about 9 orders of magnitude higher than for gas ionization. It may only take a couple of nanoseconds - a few bunch passages - until the total number of electrons per meter equals the average number of beam particles, at which point the build up saturates due to space-charge effects.

If the photoemission occurs in a dipolar magnetic field, many electrons are confined to the vicinity of the wall; they are lost quickly and neither gain significant amounts of energy nor directly harm the beam. Due to photon reflection, a fraction of the photoelectrons are emitted at the top and bottom of the chamber, however. Following the dipole field lines, they can approach the beam. These electrons as well as those created by gas ionization can initiate a beaminduced multi-pacting process based on secondary emission.

The secondary electrons consist of both true secondaries and elastically scattered or rediffused electrons. Parametrizations can be found in Refs. [16, 17, 18, 19]. Each of the two components is described by two parameters. The true secondary yield is characterized by the value of the maximum yield at perpendicular incidence, $\delta_{\max }$, 
and by the primary energy $\epsilon_{\max }$, at which the maximum yield is assumed. The yield of the elastically reflected component is maximum for primary energies near zero. It may be parametrized by its peak value, $\delta_{\mathrm{el}, E}$ and by the width of primary energies over which it extends, $\sigma_{\mathrm{el}}$.

Proper beam induced 'multipacting' occurs when electrons emitted from the wall are accelerated in the field of a passing bunch and hit the wall on the opposite side just prior to the passage of the next bunch, whose field then accelerates the secondaries. Thus, one parameter which characterizes the nature of the electron cloud effect is the ratio of the minimum electron travel time across the chamber to the bunch spacing [10]: $n_{\min }=h_{y}^{2} /\left(N_{b} r_{e} L_{\mathrm{sep}}\right)$. When $n_{\text {min }}=1$ the exact resonance condition is met. If $n_{\min }<1$, part of the primary electrons is lost before the next bunch arrives, leaving behind low-energetic secondaries. If $n_{\min }>1$, the primary electrons interact with more than 1 bunch. Table 1 compares general machine parameters and the values of $n_{\min }$ for several existing storage rings where electron clouds are observed with those for two accelerators now under construction (LHC and SNS). The table suggests that electron clouds can occur for almost any value of $n_{\min }$. We will focus on the parameter range relevant to the LHC beam at CERN and to the two B factories.

Various simulation programs have been developed which model the build up of the electron cloud in the vacuum chamber. The first program, PEI, was written by K. Ohmi at KEK in 1995 [5]; the second and third, called POSINST and ECLOUD, originated at LBNL, SLAC and CERN in 1997 [18, 9]. These programs include photoemission, gas ionization, and secondary emission, the electron electric space-charge field, the image charges of beam and electrons in an elliptical chamber, and optional external magnetic fields. For special studies, clearing electrodes, electro-magnetic waves, electron-cloud monitors, and the beam magnetic field have been introduced. Analytical approaches for computing the multipacting avalanche have also been discussed [20].

Magnitude and time constant of the real electron build up can be inferred, either directly or indirectly, from a variety of indicators, such as (1) nonlinear pressure rise, (2) tune shift along the train, (3) signals on pick-ups or dedicated electron monitors, (4) beam-size blow up along the bunch train, and (5) specific luminosity along the train.

For example, at the CERN SPS, a pressure rise is observed. The equation for the pressure balance reads $S_{\text {eff }} P /\left(k_{B} T\right)=Q$, where $S_{\text {eff }}$ denotes the pumping speed in units of cubic meter per meter per second, $Q=$ $\alpha d^{2} \lambda_{e} /(d t d s)$ the total flux of molecules per unit length $(\alpha$ is the desorption yield per electron), and $P=k_{B} T N / V$. Assuming typical values $P=100$ nTorr, $\alpha \approx 0.1$ and $S_{\text {eff }} \approx 201 \mathrm{~s}^{-1} \mathrm{~m}^{-1}$, we estimate the electron flux as [21] $d \lambda_{e} / d s=T_{r e v} /\left(\alpha k_{B} T\right) S_{\text {eff }} P \approx 10^{10} \mathrm{e}^{-}$per 81-bunch train and meter. Electron bombardment was directly observed on various detectors, on which a few $10^{8}$ electrons were deposited per bunch passage [22]; this amounted to $10^{9}-10^{10} \mathrm{e}^{-}$per train, or, with an effective pick-up length of about $10 \mathrm{~cm}$, to $d \lambda_{e} / d s \approx 10^{10} \mathrm{e}^{-}$per bunch-train and per meter, consistent with the first estimate.

Figure 1 illustrates a pick-up signal during the passage of a bunch train. The baseline of the difference signal starts shifting at the center of the train. In Fig. 2 the simulated electron build up is shown. Measurement and simulation agree best, if a significant component of elastically scattered low-energy electrons is assumed [19].

At APS and KEKB, the amount as well as the energy spectrum of electrons incident on the wall was measured by dedicated monitors [23], validating the simulations.

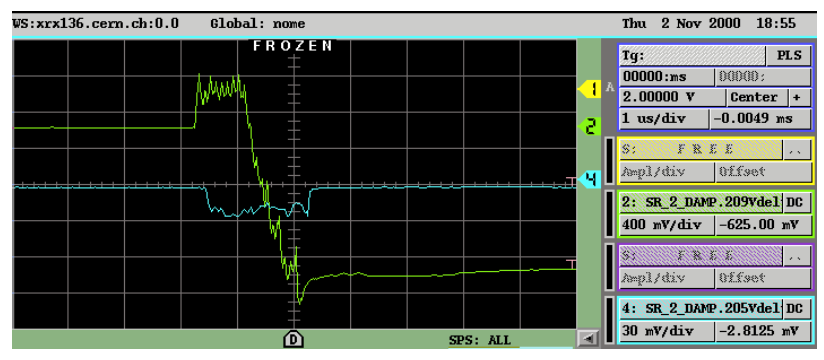

Figure 1: Sum and difference signal on damper pick-up during the passage of an LHC batch in the SPS ( $1 \mu \mathrm{s} / \mathrm{div})$. (Courtesy W. Hofle, 2001).

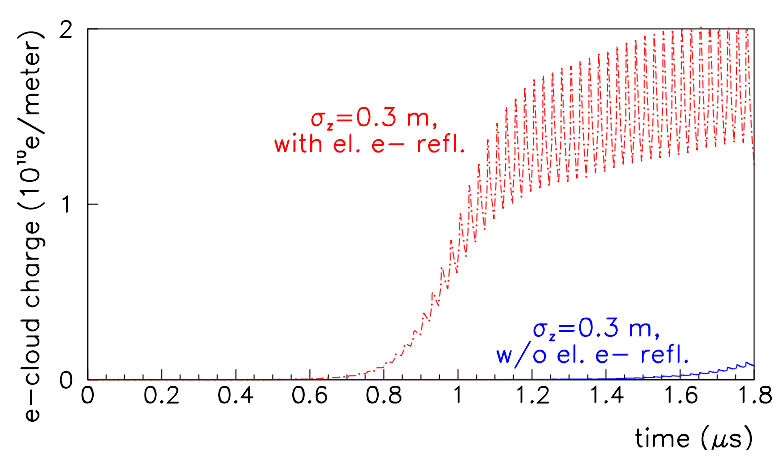

Figure 2: Simulated evolution of the electron line density in units of $\mathrm{m}^{-1}$ as a function of time in s, for an SPS dipole chamber, with and without elastic electron reflection [19].

For field-free regions and dipoles, the simulated evolution of the electron-cloud density near the beam is consistent with the measured tune shift and beam-size variation along a bunch train. However, the persistent slow blow up observed at KEKB after the installation of many solenoids $[24,25]$ was not reproduced in simulations so far.

Both at the SPS and the KEKB LER, various gaps were introduced between trains, in an attempt to identify decay time constants. A gap of $300 \mathrm{~ns}$ is clearly insufficient to reset the memory of the cloud, whereas a gap of $600 \mathrm{~ns}$ could accomplish this at both accelerators, for trains of moderate lengths.

At KEKB, two different time constants govern the decay of the cloud. In spring 2000, the tune shift of a single witness bunch injected at different distances behind a train decayed with a time constant of about $30 \mathrm{~ns}$ (25 rf 
buckets); see Fig. 3. However, the time constant relevant to bunch trains was much longer. The beam size in a first train started to blow up at the 7th bunch; by contrast already the second bunch in a subsequent train blew up for a train separation of $64 \mathrm{~ns}$ [25], i.e., twice the single-bunch decay constant. Although this measurement was performed when small quadrupole fields were introduced in large parts of the machine, it agreed with simulations for a field-free region, in which the central electron density is rapidly reestablished after the 1st bunch in the second train; see Fig. 4. This result motivated the exhaustive installation of solenoids all around the ring, which has doubled the specific luminosity [25]. With colliding beams and for train lengths of several microseconds, after the first solenoid installation, the train-to-train decay time constant seemed to exceed $2 \mu \mathrm{s}$, and, in addition, there was a hysteresis effect which increased the beam sizes after about $100 \mathrm{~s}$ [24]. These last two observations have not yet been explained by simulations.

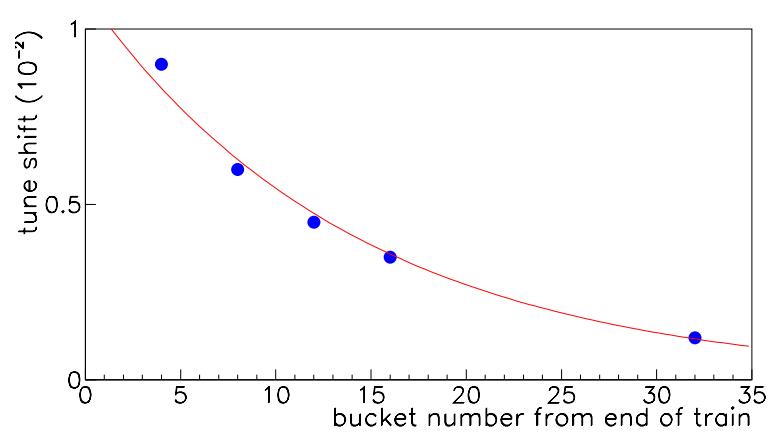

Figure 3: Tune shift of a witness bunch as a function of distance behind a bunch train [26]. (Courtesy T. Ieiri, 2001).

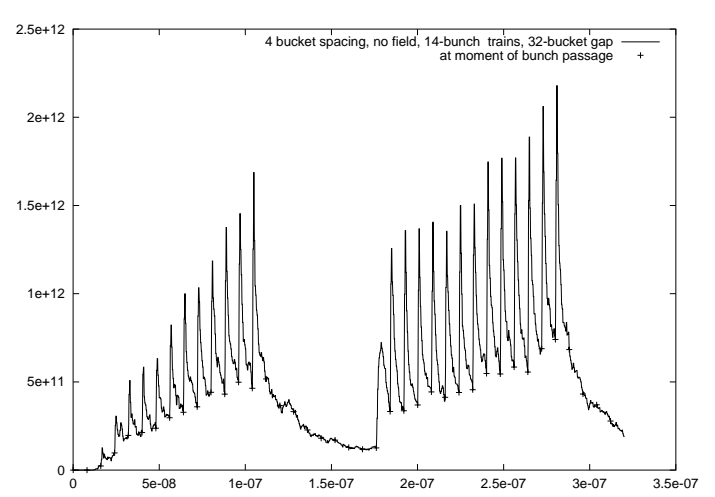

Figure 4: Simulated electron density near beam per cubic meter for a field-free region at KEKB vs. time in seconds, during the passage of two bunch trains each (4 bucket spacing) with a train-to-train gap of 32 buckets [27].

\section{WAKE FIELDS AND INSTABILITY}

If a bunch is off-set with respect to the other bunches, it will perturb the electron-cloud distribution. Similar to a multibunch wake field, the electron cloud couples the mo- tion of subsequent bunches, which gives rise to multibunch instabilities as observed at the KEK PF, BEPC, and, in the horizontal plane, at the SPS.

In the simulation, the effective wake field is computed by first establishing a stationary cloud, and then diplacing one of the bunches transversely. The kick that the disturbed electron cloud exerts on the following bunch yields an estimate of the bunch-to-bunch dipole wake field, from which the multibunch growth rate can be computed $[4,5]$. A weak-strong simulation code confirmed the results [28].

Considering a beam passing through a static electron cloud of uniform density $\rho_{e}$ in an elliptical chamber with semi axes $h_{x}$ and $h_{y}$, the coherent tune shift is $[29,19]$ $\Delta Q_{x, y}=h_{y, x} \beta_{x, y} C r_{p} \rho_{e} /\left(\gamma\left(h_{x}+h_{y}\right)\right)$, where $C$ denotes the circumference, $\beta_{x, y}$ the average beta function, and $r_{p}$ the classical particle radius. In particular, the ratio of horizontal and vertical coherent tune shifts equals the inverse of the chamber aspect ratio: $\Delta Q_{y} / \Delta Q_{x} \approx h_{x} / h_{y}$. Measured coherent tune shifts at KEKB and SPS are of the order 0.01, and consistent with simulated electron densities.

During a bunch passage, electrons are accumulated inside the beam volume [29, 27]. This induces an incoherent betaton tune spread, which can be much larger than the coherent tune shift. Possible consequences are the excitation of synchrobetatron resonances [29], as well as a change of the single-particle beta functions and dispersion. Sensitivity is high if the betatron tunes are near an integer or half integer. Introducing the electron oscillation frequency inside the bunch, $\omega_{e ; x, y} \approx c\left(2 N_{b} r_{e} /\left(\sqrt{2 \pi} \sigma_{z} \sigma_{x, y}\left(\sigma_{x}+\sigma_{y}\right)\right)\right)^{1 / 2}$, the electron density enhancement near the beam center at the end of the bunch passage is roughly given by $H_{e} \approx$ $\left(1+4 \sigma_{z} \omega_{e, x} /(\pi c)\right) \times\left(1+4 \sigma_{z} \omega_{e, y} /(\pi c)\right)$. Numbers are listed in Table 1

After a bunch passage, electrons can remain near the chamber axis if the density changes adiabatically [30]. Using the WKB approximation [31], the adiabaticity condition is $A \equiv \sigma_{z} \omega_{e, y} \sqrt{8 e} / c \gg 1$, where, exceptionally, $e=2.718 \ldots$ Most storage rings operate at $A \approx 10$ (Table 1).

If there is an initial small perturbation along the bunch, it will be amplified by the electron cloud, whose effect is then similar to a short-range wake field [32]. The Greenfunction wake field can be simulated by transversely displacing a longitudinal slice. The result can be well fitted by a broadband resonator. The resonator (electron) frequencies $\omega_{R} \approx \omega_{e}$ are of order $2 \times 10^{11} \mathrm{~s}^{-1}$ for KEKB and $1.4 \times 10^{9} \mathrm{~s}^{-1}$ for SPS, the $Q$ values vary between 1 and 6 , and the shunt impedances $c R_{S} / Q$ amount to a few $10^{6}$ $\mathrm{m}^{-2}$ [32].

Standard procedures have been applied for estimating threshold densities and instability rise times. Adapting the formalism of the fast-beam ion instability [33] to the electron-cloud problem gives a first rough estimate of the rise time [34] $1 / \tau \approx 4 \pi \rho_{e} N_{b}^{1 / 2} r_{p} r_{e}^{1 / 2} \sigma_{z}^{1 / 2} \sigma_{x} \beta c /\left(\gamma \sigma_{y}^{1 / 2}\left(\sigma_{x}+\sigma_{y}\right)^{3 / 2} \propto\right.$ $N_{b}^{3 / 2} \sigma_{z}^{1 / 2} / L_{\mathrm{sep}} / \sigma_{y}^{1 / 2}$. We have highlighted the depen- 
Table 1: Selected parameters for a few storage rings.

\begin{tabular}{|l|c|c|c|c|c|c|c|}
\hline accelerator & PEP-II & KEKB & PS & SPS & LHC & PSR & SNS \\
\hline species & $\mathrm{e}^{+}$ & $\mathrm{e}^{+}$ & $\mathrm{p}$ & $\mathrm{p}$ & $\mathrm{p}$ & $\mathrm{p}$ & $\mathrm{p}$ \\
beam energy [GeV] & 3.1 & 3.5 & 26 & 26 & 7000 & 1.64 & 2 \\
bunch population $N_{b}\left[10^{10}\right]$ & 9 & 3.3 & 10 & 10 & 10 & 5000 & 10000 \\
bunch spacing $L_{\text {sep }}[\mathrm{m}]$ & 2.5 & 2.4 & 7.5 & 7.5 & 7.5 & $(108)$ & $(248)$ \\
rms bunch length $\sigma_{z}[\mathrm{~m}]$ & 0.013 & 0.004 & 0.3 & 0.3 & 0.077 & 25 & 30 \\
rms beam sizes $\sigma_{x, y}[\mathrm{~mm}]$ & $1.4,0.2$ & $0.42,0.06$ & $2.4,1.3$ & $3,2.3$ & $0.3,0.3$ & $25,7.5$ & 0.6 \\
chamber half dimensions $h_{x, y}[\mathrm{~mm}]$ & 25 & 47 & 70,35 & $70,22.5$ & 22,18 & 50 & 100 \\
slippage factor $\eta\left[10^{-3}\right]$ & 1.3 & 0.18 & 0.026 & 0.58 & 0.35 & 188 & 36 \\
synchrotron tune $Q_{s}$ & 0.03 & 0.015 & 0.004 & 0.006 & 0.002 & 0.0004 & 0.0007 \\
circumference $C[\mathrm{~km}]$ & 2.2 & 3.0 & 0.63 & 6.9 & 27 & 0.09 & 0.22 \\
average beta function $\beta$ & 18 & 15 & 15 & 40 & 80 & 5 & 6 \\
\hline parameter $n_{\min }$ & 1 & 10 & 0.58 & 0.24 & 0.15 & 0.0002 & 0.0001 \\
$\mathrm{e}^{-}$oscillations/bunch $n_{\mathrm{osc}} \equiv \omega_{e} \sigma_{z} /(\pi c)$ & 0.9 & 1.0 & 1 & 0.75 & 3 & 34 & 970 \\
density enhancement $H_{e}$ & 12 & 13 & 26 & 14 & 190 & $\left(10^{5}\right)$ & $\left(10^{7}\right)$ \\
adiabaticity $A$ & 8 & 9 & 11 & 7 & 28 & 300 & 8600 \\
TMCI threshold $\rho_{e}\left[10^{12} \mathrm{~m}^{-3}\right]$ & 1 & 0.5 & 5 & 0.25 & 3 & 0.6 & 0.5 \\
density ratio $\rho_{e, \text { sat }} / \rho_{e, \text { thresh }}$ & 19 & 4 & 0.35 & 11 & 4 & 92 & 27 \\
\hline
\end{tabular}

dence on $N_{b}, L_{\mathrm{sep}}, \sigma_{y}$ and $\sigma_{z}$, assuming that the electron density scales with the average 'neutralization' density, $\rho_{e, \mathrm{sat}} \approx N_{b} /\left(\pi L_{\mathrm{sep}} h_{x} h_{y}\right)$.

Using a two particle model, where the first particle has a finite length, the wake field acting on the trailing particle is estimated as [35] $W_{y, 0} \approx 8 \pi \rho_{e} C / N_{b}$ for sufficiently long bunches, i.e., $\sigma_{z} \omega_{e}>c \pi / 2$. Assuming that the electron density saturates near the neutralization density $\rho_{e, \text { sat }}$, the wake field can be rewritten as $W_{y, 0} \approx 8 C /\left(L_{\text {sep }} h_{x} h_{y}\right)$, which depends only on geometric quantities. The associated beam break-up growth rate, e.g., in a transport line or isochronous ring, is $1 / \tau_{y, \mathrm{BBU}} \approx 2 \pi \rho_{e} r_{p} c \beta / \gamma \propto N_{b} / L_{\text {sep }}$. For shorter bunches, i.e., $\sigma_{z} \omega_{e}<c \pi / 2$, the wake reads $W_{y, 0} \approx 4 \pi \rho_{e} C r_{p} \sigma_{z} /\left(\left(\sigma_{x}+\sigma_{y}\right) \sigma_{y}\right)$, and $1 / \tau_{y, \mathrm{BBU}} \propto$ $N_{b}^{2} \sigma_{z} / L_{\mathrm{sep}} / \sigma_{y}$. In a ring with synchrotron oscillation, the beam break up manifests itself either as a head-tail or strong head-tail (TMCI) instability. For positive chromaticities $Q^{\prime}=\Delta Q_{\beta} /(\Delta p / p)$, the higher-order head-tail modes are unstable. For 'long' bunches, the growth rate of the $l=1$ mode is $1 / \tau_{y, \text { ht }}=64 Q_{y}^{\prime} \rho_{e} \beta_{y} r_{p} \sigma_{z} /\left(3 T_{0} \eta \gamma\right) \propto$ $N_{b} \sigma_{z} /\left(L_{\mathrm{sep}} \eta\right)$, and the threshold electron density for the TMCI instability $\rho_{e, \mathrm{thr}} \approx 2 \gamma Q_{s} /\left(\pi \beta_{y} r_{p} C\right)$, where $T_{0}$ denotes the revolution period, and $Q_{s}$ the synchrotron tune. Since $\rho_{e \text {,sat }} \propto N_{b} / L_{\text {sep }}$, this implies the scaling $N_{b, \text { thr }} \propto$ $L_{\text {sep }}$. Threshold densities are listed in Table 1.

Various macroparticle computer simulations of the single-bunch instability were performed. The first simulation tracked micro-bunches with finite transverse size distributed as a multiple air bag in synchrotron phase space [35]. Another approach calculates the force on the electrons using a soft-Gaussian approximation for the beam distribution [36]. Latest simulations employ particle-incell codes [37, 38]. Simulated thresholds roughly confirm the analytical estimates [35].

In parallel, analytical approaches based on perturbative treatments of the Vlasov equation have been pursued. Ap- proximating the wake by a broadband resonator, Ref. [32] calculates the threshold electron density for mode coupling (TMCI) and for fast blow up [39]. The results are consistent with the macro-particle simulations.

Observations at KEKB indicate that the threshold current of the blow up scales with $N_{b} / L_{\text {sep }}$, i.e., it varies in proportion to the electron density, whereas the beam size above the threshold scales with $N_{b}^{2} / L_{\text {sep }}$, at least during injection [24]. The single-bunch characteristics of the blow up was confirmed by varying the charge of a single witness bunch at the end of a train, keeping the current of all preceding bunches constant. The beam size of the test bunch increased when its charge was increased [25].

Similar observations were made at the SPS, where the most intense bunches are the first to be lost. Here, direct evidence for a head-tail motion inside the bunch comes from a wide-band pick up, which samples the beam motion every $0.5 \mathrm{~ns}$ (compared with a bunch length of $4 \sigma_{z} \approx 3 \mathrm{~ns}$ ) for 56 consecutive turns. In the vertical plane the head-tail motion appears to be random from bunch to bunch, and there is evidence for motion at frequencies around $800 \mathrm{MHz}$ [40]. The interaction length of the vertical electron-cloud wake field could be reconstructed from the monitor data [41]. It is about $0.3-0.5$ of the total bunch length, consistent with the expected electron oscillation frequency inside the bunch. In the horizontal plane the detector shows coupled-bunch motion, indicating that the electron cloud acts like a longrange wake field covering one or more bunch spacings.

The electron cloud will also generate a longitudinal wake field, and thus may give rise to potential-well distortion and eventually longitudinal microwave instability. Figure 5 shows simulated oscillations of the longitudinal electric field excited by a bunch passing through an electron-cloud plasma [38]. 


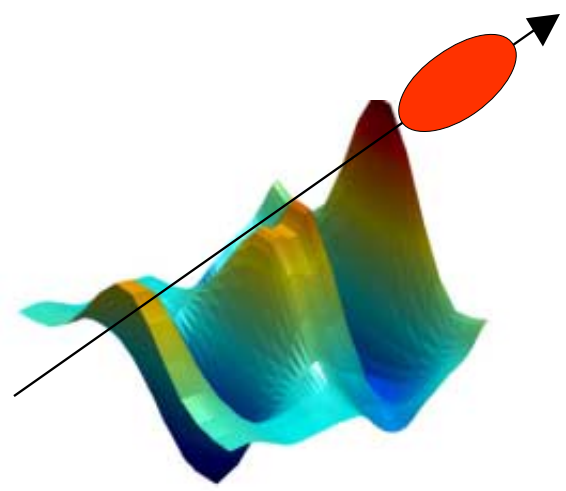

Figure 5: Longitudinal electric field for a bunch passing through an electron cloud. (Courtesy S. Lee, 2001).

\section{CONCURRING ISSUES}

The heat load deposited by the electron cloud on the beam screen and cold bore of the superconducting magnets is a major concern for the LHC $[11,19]$, and will determine the choice of bunch fill patterns during the commissioning.

The combined effect of electron cloud and conventional impedance is under investigation [36]. Preliminary evidence at KEKB and PEP-II suggests that the electron-cloud instability aggravates the beam-beam effect. This is expected as both give rise to a large tune spread and to pseudo head-tail wake fields. In addition, the blow up of one beam can induce a beam-beam flip-flop state, observed at both machines. Simulation suggest that ion accumulation inside the cloud is not significant [42]. Also negligible is the absorption of synchrotron radiation by the electrons [43].

Collective plasma effects likely become important, however, since the simulated fields exceed by a factor of 10 the maximum wave amplitude - about $100 \mathrm{kV} / \mathrm{m}$ - supported by the electron-cloud plasma ('cold wavebreaking') $[44,38]$. An unresolved question is whether the combination of dc magnetic (dipoles) and ac electric fields (beam) can produce a 'magnetron effect' [45].

\section{CURES}

The number of primary or secondary electrons can be reduced by an antechamber, which absorbs most of the photons (PEP-II), by surface coating for minimum secondary emission yield, e.g., with TiN, (PEP-II, PSR, LHC), by a sawtooth chamber for minimum photon reflection (LHC), or by changing the bunch length. Long-term bombardment with cloud electrons will further reduce the secondary emission yield. First evidence for this 'surface scrubbing' was seen at the SPS and at KEKB. The memory of the scrubbing may be preserved by $\mathrm{N}_{2}$ glow discharge [46].

The flow of electrons towards the beam can be suppressed or modified by magnetic fields, such as weak solenoids (KEKB, PEP-II), or by clearing electrodes (ISR). Special bunch filling patterns minimize the average central electron density and optimize luminosity (PEP-II, KEKB, LHC). Intermediate lower-charge 'satellite' bunches may clear the beam pipe from electrons before the next main bunch arrives $[47,30,19]$.
Instability thresholds can be raised by Landau-damping octupoles (KEK PF, BEPC), by a large chromaticity (BEPC, SPS, KEKB), by a dedicated TMCI feedback as used for conventional instabilities at VEPP-4M [48], by detuning the lattice, and by optimizing the bunch length.

\section{ACKNOWLEDGEMENTS}

I thank G. Arduini, R. Cappi, F. Caspers, I. Collins, K. Cornelis, F.-J. Decker, H. Fukuma, M. Furman, M. Giovannozzi, N. Hilleret, W. Höfle, T. Katsouleas, T. Ieiri, D. Kaltchev, S. Lee, E. Metral, K. Ohmi, K. Oide, M. Pivi, M. Plum, B. Richter, A. Rossi, F. Ruggiero, and G. Rumolo for discussions and informations.

\section{REFERENCES}

[1] H. Hereward, CERN 71-15; E. Keil, B. Zotter, CERN-ISRTH-71-58 (1971).

[2] O. Gröbner, HEACC'77, Protvino (1977).

[3] D. Neuffer, et al., Part. Acc. 23 (1988); NIM A 321 (1992).

[4] M. Izawa, et al., Phys. Rev. Lett. 74, 5044 (1995).

[5] K. Ohmi, Phys. Rev. Lett. 75, 1526 (1995).

[6] Z.Y. Guo et al., APAC 98, Tsukuba (1998).

[7] M. A. Furman, et al., PAC97, Vancouver (1997).

[8] S. Heifets, SLAC-PUB-6956 (1995).

[9] F. Zimmermann, LHC PR 95, SLAC-PUB-7425 (1997).

[10] O. Gröbner, PAC97, Vancouver (1997).

[11] O. S. Brüning, CERN LHC PR 158 and EPAC98 (1997).

[12] M. Furman, LHC Project Report 180 (1998).

[13] T. Holmquist, J. Rogers, Phys. Rev. Lett. 79, 3186 (1997).

[14] E. Metral, et al., these proceedings.

[15] M. Sands, SLAC-0121 (1970).

[16] H. Seiler, J. Appl. Phys. 54 (11) (1983).

[17] R. Kirby, et al., SLAC-PUB-8212 (2000).

[18] M.A. Furman, et al., KEK Proc. 97-17, p. 170 (1997).

[19] F. Zimmermann, Chamonix X \& XI, CERN-SL-2000-007 \& 2001-003-DI; G. Rumolo et al., PRST-AB 012801 (2001).

[20] G. Stupakov, CERN LHC PR 141 (1997); L. Vos, CERN LHC PN 150 (1998).

[21] O. Gröbner, private communication (1999).

[22] W. Höfle, Chamonix X, CERN-SL-2000-007-DI (2000).

[23] K. Harkay, et al., PAC99 Washington; Y. Ohnishi, et al., HEACC2001 Tsukuba (2001).

[24] K. Oide, Chamonix XI, CERN-SL-2000-007 DI (2001).

[25] H. Fukuma, et al., HEACC'01 Tsukuba (2001); H. Fukuma, et al., EPAC2000 Vienna (2000); H. Fukuma and K. Ohmi, private communications (2001).

[26] T. Ieiri, et al., HEACC01 Tsukuba (2001).

[27] F. Zimmermann, et al., CERN-SL-2000-017 (AP); F. Z., CERN-SL-Note-2000-061 AP (2000).

[28] K. Ohmi, PAC97 Vancouver (1997).

[29] M.A. Furman, et al., PAC99 Washington (1999).

[30] B. Richter, SLAC memo, unpublished (2000).

[31] J. Liouville, J. de Math. 2, 16, 418 (1837).

[32] K. Ohmi, et al., HEACC'01 Tsukuba (2001).

[33] T. Raubenheimer, et al., Phys. Rev. E52, 5487 (1995).

[34] F. Zimmermann, CERN-SL-Note-2000-004 AP (2000).

[35] K. Ohmi, F. Zimmermann, Phys. Rev. Lett. 85, 3821 (2000).

[36] G. Rumolo, et al., these proceedings.

[37] K. Ohmi, these proceedings.

[38] S. Lee, T. Katsouleas, private communication (2001).

[39] R.D. Ruth, J. Wang, IEEE Tr. NS-28, no. 3 (1981); P. Kernel, et al., EPAC 2000, Vienna (2000).

[40] G. Arduini, et al., these proceedings.

[41] K. Cornelis, Chamonix XI, CERN-SL-2001-003-DI.

[42] G. Rumolo, et al., CERN-SL-2001-014 AP (2001).

[43] D. Kaltchev, private communication (2001).

[44] J.M. Dawson, Phys. Rev. 113 (1959); T. Katsouleas, W. Mori, PRL 61, p. 90 (1988).

[45] F. Caspers, private communication (1999).

[46] J.M. Jimenez, Chamonix XI, CERN-SL-2001-003-DI.

[47] F. Ruggiero, et al., BNL, AIP Conf. Proc. 496 (1999).

[48] G. Karpov, et al., DIPAC99 (1999). 